\title{
Genetic Alterations in the Molecular Subtypes of Bladder Cancer: Illustration in the Cancer Genome Atlas Dataset
}

\author{
Woonyoung Choi $^{a, b}$, Andrea Ochoa ${ }^{a, b}$, David J. McConkey ${ }^{a, b, *}$, Mattias Aine $^{c}$, Mattias Höglund $^{c}$, \\ William Y. Kim ${ }^{d}$, Francisco X. Real ${ }^{e, f}$, Anne E. Kiltie ${ }^{g}$, Ian Milsom ${ }^{h}$, Lars Dyrskjøt $^{i}$, \\ Seth P. Lerner ${ }^{j}$
}

${ }^{a}$ Department of Urology, U.T. M.D. Anderson Cancer Center, Houston, Texas, USA; ${ }^{\mathrm{b}}$ Department of Cancer Biology, U.T. M.D. Anderson Cancer Center, Houston, Texas, USA; ${ }^{\mathrm{C}}$ Division of Oncology and Pathology, Department of Clinical Sciences, Faculty of Medicine, Lund University, Lund, Sweden; ${ }^{\mathrm{d}}$ University of North Carolina, Chapel Hill, North Carolina, USA; ${ }^{\mathrm{e}}$ Epithelial Carcinogenesis Group, Spanish National Cancer Research Centre-CNIO, Madrid, Spain; ${ }^{\mathrm{f}}$ Departament de Ciències Experimentalsi de la Salut, Universitat Pompeu Fabra, Barcelona, Spain; ${ }^{\mathrm{g}}$ Department of Oncology, University of Oxford, Oxford, UK; ${ }^{\mathrm{h}}$ Institute of Clinical Sciences, Sahlgrenska Academy at Gothenburg University, Gothenburg, Sweden; ${ }^{\mathrm{i}}$ Department of Molecular Medicine, Aarhus University Hospital, Aarhus, Denmark; ${ }^{\mathrm{j}}$ TCGA Analysis Working Group, Baylor College of Medicine, Houston, Texas, USA

\section{Article info}

\section{Article history:}

Accepted March 6, 2017

Associate Editor:

James Catto

Keywords:

Molecular subtypes

Muscle-invasive bladder cancer

DNA alterations

\begin{abstract}
Context: Recent whole genome mRNA expression profiling studies revealed that bladder cancers can be grouped into molecular subtypes, some of which share clinical properties and gene expression patterns with the intrinsic subtypes of breast cancer and the molecular subtypes found in other solid tumors. The molecular subtypes in other solid tumors are enriched with specific mutations and copy number aberrations that are thought to underlie their distinct progression patterns, and biological and clinical properties. objective: The availability of comprehensive genomic data from The Cancer Genome Atlas (TCGA) and other large projects made it possible to correlate the presence of DNA alterations with tumor molecular subtype membership. Our overall goal was to determine whether specific DNA mutations and/or copy number variations are enriched in specific molecular subtypes.

Evidence: We used the complete TCGA RNA-seq dataset and three different published classifiers developed by our groups to assign TCGA's bladder cancers to molecular subtypes, and examined the prevalence of the most common DNA alterations within them. We interpreted the results against the background of what was known from the published literature about the prevalence of these alterations in nonmuscle-invasive and muscle-invasive bladder cancers.

Evidence synthesis: The results confirmed that alterations involving RB1 and NFE2L2 were enriched in basal cancers, whereas alterations involving FGFR3 and KDM6A were enriched in luminal tumors.

Conclusions: The results further reinforce the conclusion that the molecular subtypes of bladder cancer are distinct disease entities with specific genetic alterations.

Patient summary: Our observation showed that some of subtype-enriched mutations and copy number aberrations are clinically actionable, which has direct implications for the clinical management of patients with bladder cancer.

(C) 2017 European Association of Urology. Published by Elsevier B.V. All rights reserved.

* Corresponding author. Johns Hopkins Greenberg Bladder Cancer Institute, 600 Wolfe Street, Marburg 149, Baltimore, MD 21287-2101, USA. E-mail address: djmcconkey@jhmi.edu (D.J. McConkey).
\end{abstract}




\section{Introduction}

Recent whole genome mRNA expression profiling studies revealed that bladder cancers can be grouped into molecular subtypes, some of which share clinical properties and gene expression patterns with the intrinsic subtypes of breast cancer and the molecular subtypes found in other solid tumors. The molecular subtypes in other solid tumors are enriched with specific mutations and copy number aberrations (CNAs) that are thought to underlie their distinct progression patterns, and biological and clinical properties.

\section{Evidence acquisition}

We used the complete The Cancer Genome Atlas (TCGA) RNA-seq dataset and three different published classifiers developed by our groups to assign TCGA's bladder cancers to molecular subtypes, and examined the prevalence of the most common DNA alterations within them (Supplementary material). We interpreted the results against the background of what was known from the published literature about the prevalence of these alterations in nonmuscle-invasive and muscle-invasive bladder cancers.

\section{Evidence synthesis}

\subsection{Clinical issues in bladder cancer}

Clinical experience and emerging genomic data support the idea that bladder cancers progress along two largely nonoverlapping tracks ("papillary" and "nonpapillary") that pose distinct challenges for clinical management [13]. Most nonmuscle-invasive bladder cancers (NMIBCs) belong to the papillary pathway and are characterized by the presence of activating type- 3 receptor for fibroblast growth factor (FGFR3) mutations, downstream Ras pathway activation, wild-type TP53, and stable genomes [1-3]. Clinically, papillary NMIBCs are rarely lethal but recur almost always, necessitating that patients receive lifelong surveillance; the repeated surgical procedures required to deal with recurrences cause significant anxiety, discomfort, and morbidity, making bladder cancer the most expensive tumor on a per patient basis. A significant proportion of cases (15-20\%) of NMIBCs progress to become muscle invasive [1,2]. However, currently no reliable tools are available to identify them before they become life threatening. The nonpapillary pathway is characterized by loss-of-function mutations and CNAs involvingTP53 and $R B 1$ and genomic instability [1,2]. It gives rise to aggressive, muscle-invasive bladder cancers (MIBCs), representing approximately $20-25 \%$ of all bladder cancers and causing death in approximately half of affected patients. Carcinoma in situ (CIS) is generally considered to be the precursor lesion for nonpapillary MIBCs [1,2], but comprehensive genomic data for CIS are not yet available, so this assumption awaits direct experimental validation. Patients with either high-grade papillary nonmuscle-invasive disease or CIS are currently treated with the same adjuvant therapy (intravesical Bacillus Calmette-Guerin [BCG] immunotherapy), but it is by no means clear that BCG produces comparable benefit in CIS and high-grade papillary tumors [1,2]. Many high-grade papillary tumors ultimately become BCG unresponsive, so clinicians are then faced with the dilemma of whether to continue using a bladder-sparing regimen or to employ definitive surgery. The latter is certainly too aggressive for those patients whose tumors could be controlled by local therapy, but again there are no reliable tools to distinguish the tumors that have the potential to metastasize from those that do not. Muscle-invasive disease is managed with definitive local therapy (chemoradiation) or surgery (cystectomy) with or without perioperative systemic cisplatin-based chemotherapy to treat subclinical metastatic disease, but it is still not possible to distinguish the patients who warrant chemotherapy from those who will not benefit from it. It would also be tremendously useful to have biomarkers that would enable patients and their physicians to choose between bladder-sparing regimens such as chemoradiation and cystectomy. Overall, it is hoped that by understanding the molecular mechanisms that give rise to papillary and nonpapillary bladder cancers, it will be possible to develop methods to inform clinical decision making at every step of disease progression and management.

\subsection{Intrinsic subtypes of cancer}

The widespread use of genomics to investigate cancer heterogeneity is transforming our understanding of cancer biology. A pioneering study in leukemia demonstrated that mRNA expression profiling could be used to distinguish ALL from AML with a high degree of accuracy [4], and a subsequent study used gene expression profiling to identify two previously unrecognized molecular subtypes of diffuse large B-cell lymphoma [5]. Importantly, patients whose tumors belonged to one of the subtypes ("germinal centerlike DLBCL") had better clinical outcomes than patients with the other ("activated B-like DLBCL") [5]. Parallel studies in breast cancer revealed that they could also be grouped into "intrinsic subtypes" that had very different biological properties and behaved clinically as distinct disease entities $[6,7]$. Patients with basal-like or HER2-enriched breast tumors had poor clinical outcomes in the absence of systemic therapy, but many of them benefited greatly from neoadjuvant chemotherapy (NAC) $[8,9]$. Patients with HER2-enriched tumors also obtained significant clinical benefit from ERBB2 antagonists [10]. In the absence of perioperative chemotherapy, women with luminal tumors had better prognoses [11] and, when given perioperative chemotherapy, most patients also obtained little to no benefit $[8,12]$. Rather, they obtained major chemopreventive clinical benefit from adjuvant therapy with selective estrogen receptor modulators (SERMs), which reduced disease recurrence by about 50\% [11]. In contrast, SERMs produced no benefit in patients with basal-like or HER2enriched tumors [11]. Subsequent studies identified molecular subtypes in head and neck squamous cell carcinomas (SCCs) [13], glioblastomas [14], and pancreatic cancers [15], 
and more recent studies are characterizing molecular subtypes that cut across cancer types [16]. The potential clinical significance of the molecular subtypes of these cancers is being investigated.

\subsection{DNA alterations in bladder cancers}

The recently completed TCGA MIBC (BLCA) project produced a comprehensive, open-access catalog of DNA alterations in a cohort of over 400 MIBCs. The first TCGA "bladder cancer study" reported the results of a comprehensive integrated genomic analysis of 131 tumors [17], and a recent review article provided an update on 238 tumors [18]. These initial results were also incorporated into a pan-cancer analysis that compared the genomic features of 12 different types of cancers [16]. Furthermore, a thorough review of the different genomic alterations that characterize low-grade papillary tumors (Ta) and MIBCs was also published recently [1]. Finally, a recent paper reported the comprehensive transcriptional analysis of a cohort of 460 patients with NMIBC [19]. Therefore, excellent, comprehensive summaries of the major genomic alterations in the complete spectrum of bladder cancers can be found elsewhere. The key findings will now be summarized briefly.

\subsubsection{Major drivers of mutagenesis}

Cigarette smoking is an established risk factor for bladder cancer [2], and chronic exposure to cigarette smoke-like nitrosamines (ie, BBN) causes bladder cancer in rodents [20]. Aromatic compounds in cigarette smoke produce DNA damage, so it was expected that a history of cigarette smoking would be associated with specific tobacco-related DNA mutations in TCGA exome sequencing data. Analyses of the initial TCGA cohort of 131 tumors failed to identify such signatures, although tumors from smokers were enriched with specific DNA methylation patterns [17]. Interestingly, a significant number of bladder cancers contained mutations in NFE2L2 (NRF2) and TXNIP [18] genes, which encode proteins that inhibit the damaging effects of the reactive oxygen species that are produced in response to cigarette smoke carcinogens. Although there was no obvious relationship between the NFE2L2 and/or TXNIP alterations and smoking status in bladder cancers, mutations in these genes were enriched in lung and head and neck cancers from smokers [21,22], suggesting potential causal roles in carcinogenesis and/or tumor progression. A more recent reanalysis of the original TCGA cohort identified a novel DNA mutational signature associated with inactivating mutations in the gene encoding the nucleotide excision repair protein, ERCC2, and established that these signatures were enriched in tumors from smokers [23]. Importantly, the signature was much more strongly associated with ERCC2 inactivation than it was with smoking, suggesting that the former was the driving force underlying the signature [23].

The APOBEC family of antiviral enzymes promotes cytosine deamination and mutagenesis of single-stranded DNA and mRNAs. Among the APOBEC genes, APOBEC3B appears to be most commonly overexpressed in solid tumors, and bladder cancers stand out for expressing some of the highest levels of APOBEC3B among all solid malignancies [24]. Aside from being upregulated by infection, APOBEC3B activity can also be increased by chemical carcinogens, which promote APOBEC3B-mediated mutagenesis by inducing the formation of the single-strand DNA intermediates that are formed during DNA damage and repair [24]. Analyses of mutational patterns have revealed that a large proportion of the total mutational burden in bladder cancer is attributable to APOBEC3B-mediated mutagenesis $[17,24,25]$, and the prevalence of APOBEC3Bassociated mutations increased with subclonal evolution in lung cancers $[24,26]$. Furthermore, it was recently found that an APOBEC mutation signature was significantly enriched in high-risk NMIBCs [19]. Taken together, accumulating data suggest that APOBEC-mediated mutations may play a central causative role in driving bladder cancer genomic heterogeneity and disease progression.

\subsubsection{Major targets of DNA alterations}

Histone modifications play central roles in the regulation of gene expression, and whole exome sequencing studies revealed that mutations in chromatin-modifying enzymes were extremely common in bladder cancers [27]. Among them, inactivating mutations in the histone $\mathrm{H} 3$ lysine 27 (H3K27) demethylase KDM6A (also known as UTX) were most common and enriched in NMIBCs (32-43\%) [18,27], whereas inactivating mutations in the SET family histone H3 lysine 4 (H3K4) methyltransferase MLL2 were more common in MIBCs (19\%) [18], and mutations in KDM6A and MLL2 were mutually exclusive [18]. Although the biological consequences of these events have not been defined experimentally, they would be expected to lead to decreased RNA polymerase accessibility, gene silencing, and a less well-differentiated phenotype. In-depth chromatin immunoprecipitation/sequencing (ChIP-seq) studies are required to directly address this hypothesis.

As introduced above, one of the most striking differences between NMIBCs and MIBCs is the relative frequency of TP53 gene inactivation and relative levels of genomic instability. Overall, mutations in TP53 were observed in about $50 \%$ of MIBCs but were less common in NMIBCs (20\% of tumors) [1,28-30]. Interestingly, $85 \%$ of high-grade NMIBCs (T1G3) contained p53 pathway alterations [31]. Furthermore, amplification of TP53's inhibitor, MDM2, occurred in approximately $9 \%$ of MIBCs [1,18], indicating that TP53 inactivation occurred in the majority of muscle-invasive tumors. RB1 inactivation was also much more common in MIBCs as compared with that in NMIBCs [18], and mutations in RB1 tended to be associated with mutations in TP53 [18]. Interestingly, the same patterns were not observed with RB1's upstream inhibitor (CDKN2A), which was deleted in approximately equal numbers of NMIBCs and MIBCs (50\%) [1]. Dysregulation of other genes that promote cell cycle progression was also common in bladder cancers. Amplification of cyclin D1 was reported in approximately $20 \%$ of NMIBCs and MIBCs [1], and amplification of E2F3 was observed in high-grade T1 lesions and MIBCs [1,32]. An early study reported that MYC amplification was associated with 
an advanced stage and grade [33], and in more recent studies, MYC amplification was observed in about $13 \%$ of MIBCs [18].

Inactivating mutations in genes encoding DNA repair proteins were also relatively common in MIBCs [17,34]. The most prevalent were inactivating mutations in ERCC2 (12\% of tumors) [18], which were linked to sensitivity to neoadjuvant cisplatin-based combination chemotherapy [35]. Inactivating mutations in several other DNA repair proteins were also linked to cisplatin sensitivity [36].

Inactivating mutations in STAG2, a component of the cohesin complex that functions in chromosome segregation, were common in NMIBCs and MIBCs [34,37-40], but their biological significance remains unclear; the canonical role of the cohesin complex would suggest that inactivation of STAG2 should produce genomic instability, but the significant enrichment of STAG2 mutations in low-grade tumors that largely lack aneuploidy [40] argues against this being their most relevant effect in bladder cancers [34,3740]. Alternative mechanisms include alterations in highorder chromatin organization and gene expression.

Activating mutations in the telomerase (TERT1) promoter were common in both NMIBCs and MIBCs [41-48], making them attractive biomarkers for early detection of recurrence $[44,45]$ and potentially as therapeutic targets across the course of disease progression.

Bladder cancers often contained DNA alterations involving oncogenes or tumor suppressor genes that regulate activation of the Ras-MEK-ERK and PI3 kinase-AKT-mTOR pathways. These pathways control progression through the RB1-dependent G1-S cell cycle restriction point, anabolic metabolism, and cell survival. Activating mutations in FGFR3 were detected in up to $80 \%$ of NMIBCs and approximately $15-20 \%$ of MIBCs, consistent with earlier studies $[1,49,50]$. Preclinical studies demonstrated that these FGFR3 mutations, which cause constitutive receptor activation, functioned to promote proliferation via downstream activation of the ERKs [51,52]. Papillary and nonpapillary cancers contained similar frequencies of activating RAS mutations (5-10\%) [51], which also function to promote downstream ERK activation. RAS and FGFR3 mutations occurred in a mutually exclusive fashion [18], so together they probably accounted for enhanced ERK activation in almost $90 \%$ of NMIBCs. Although activating FGFR3 mutations were less common in MIBCs, some MIBCs contained activating FGFR3 fusions [53]. They also contained activating mutations, fusions, or amplification of genes encoding members of the epidermal growth factor receptor (EGFR) family [17], including the EGFR itself (about $10 \%$ of tumors), ERBB2 (about $10 \%$ of tumors), ERBB3 (about $10 \%$ tumors), and ERBB4 (about $6 \%$ of tumors). Other tumors contained inactivating mutations in the RAS inhibitor, NF1 (over 10\%) [1,18]; so, together, these alterations probably promoted RAS pathway activation in over 50\% of MIBCs. With respect to the PI3 kinase/AKT pathway, activating PIK3CA mutations-predominantly in the region coding for the helical domain, probably caused by APOBEC3B-mediated mutagenesis [24]-were common in both NMIBCs and MIBCs. Amplification of AKT3 or inactivating mutations in various negative regulators of the PI3 kinase/AKT/mTOR pathway (including the lipid phosphatase PTEN) were also observed, leading to predicted pathway activation in almost $75 \%$ of MIBCs $[1,18]$.

Mutations and/or amplification of transcription factors implicated in urothelial terminal differentiation were found in MIBCs [18]. Amplifications of peroxisome proliferator activator receptor-gamma (PPARG), GATA3, and SOX4 were frequently detected, occurring in about $10-15 \%$ of tumors [18]. Mutations in ELF3, RXRA, and KLF5 were also relatively common, occurring in 5-10\% of tumors [18]. Mutations in FOXA1 were observed in about $5 \%$ of tumors, and deletion of FOXQ1 occurred in about $10 \%$ of tumors [18]. Inactivating mutations in NOTCH1 and NOTCH2 have also been reported in MIBCs [54], and preclinical studies in mouse models suggested that they promoted tumor progression by facilitating epithelial-to-mesenchymal transition (EMT) [55]. Finally, mutations in the ubiquitin ligase and NOTCH pathway regulator, FBXW7, occurred in about 7\% of MIBCs [18]. Although inactivating mutations in other developmental pathways were less common, preclinical studies have suggested that activation of the $\mathrm{Wnt} / \beta$-catenin pathway and downregulation of the sonic hedgehog pathway also contribute to bladder cancer progression [56,57].

\subsection{Molecular subtypes of bladder cancer}

The identification and validation of molecular subtypes in other malignancies provided the impetus to use transcriptome profiling to search for molecular subtypes in bladder cancers. The initial results established that unsupervised analyses of gene expression could distinguish most NMIBCs from most MIBCs [58-60]. Furthermore, early studies of NMIBCs identified gene expression signatures associated with disease aggressiveness using unsupervised analyses [61-63]. These studies showed the first indications of the presence of major molecular subtypes in bladder cancer $[19,64]$. One of our groups (M.H., Lund University, Lund, Sweden) extended these findings by implicating differences in TP53 mutation frequencies and/or genomic instability to the formation of these two major gene expression subtypes [28]. Subsequently, they used a large cohort of NMIBCs and MIBCs $(n=308)$ to identify additional subtypes within the two major clusters [65]. The Lund classification revealed that bladder cancers could be segregated into at least five molecular subtypes, termed urobasal A (uroA), urobasal B (uroB), genomically unstable (GU), infiltrated, and SCC like (SCCL) [65]. The uroA and uroB tumors were characterized by stratified expression of differentiation-associated biomarkers reminiscent of what is observed in the normal urothelium, whereas differentiation-associated biomarkers displayed abnormal expression in the GU and SCCL tumors. The SCCL subtype was characterized by expression of squamous keratins (KRT5, KRT6, and KRT14) and keratinization-associated genes [66], and the SCCL and uroB tumors were both enriched with various degrees of squamous differentiation markers $[65,66]$. As its name implies, the infiltrated subtype was characterized by the expression of 
biomarkers characteristic of immune and other stromal cells.

Analysis of a large cohort of 460 tumors recently identified three distinct subtypes (Classes 1-3) in NMIBC [19] The study validated previously observed subgroups in NMIBC and identified a new subtype (Class 3 ) that showed basal-like characteristics and pronounced expression of lncRNAs. Mutations were called from RNA-seq data, which have potential limitations. Frequent mutations were observed in genes encoding proteins involved in chromatin organization and cytoskeletal functions. Furthermore, highrisk tumors (Class 2) were enriched for mutations in, for example, TP53 and ERBB2, and for APOBEC-related mutations. The observation of enrichment for APOBEC-related mutations in high-risk NMIBCs indicates that APOBEC may drive disease progression in NMIBC.

Other groups performed independent studies to identify molecular subtypes in cohorts of MIBC $[17,67,68]$. A group led by one of us (W.K., University of North Carolina [UNC], Chapel Hill, NC, USA) assembled a meta-dataset of 262 high-grade tumors from four previously published cohorts for discovery and created a new dataset from 49 tumors collected at Memorial Sloan Kettering Cancer Center for validation. Using consensus clustering, they identified two molecular subtypes of MIBC in both datasets [68]. The genes that distinguished the two clusters had previously been implicated in urothelial differentiation and overlapped substantially with the genes that distinguished the basal-like and luminal intrinsic subtypes of breast cancer, leading the UNC investigators to name their MIBC subtypes "basal like" and "luminal" [68]. In parallel, another of our groups (W.C. and D.J.M., University of Texas MD Anderson Cancer Center, Houston, TX USA) created two whole transcriptome datasets ( $n=73$ and $n=57$ tumors) and used unsupervised hierarchical clustering to identify three candidate subtypes [67]. The MD Anderson group also noted that the genes that characterized two of the subtypes were similar to the ones that distinguished basal-like and luminal breast cancers, prompting them to term them "basal" and "luminal" [67]. The group's third subtype was distinguished from the other two by stromal biomarkers and an active $p 53$ gene expression signature, so they termed it "p53like" [67]. Finally, TCGA used a combination of different approaches to identify four molecular subtypes in an RNAseq dataset generated from 129 tumors [17]. Although TCGA discussed whether or not to name them "basal" and "luminal" in the first marker paper, they settled on a more conservative approach and termed them "clusters I-IV" but discussed their similarities to the intrinsic subtypes of breast cancer [17]. In addition, TCGA noted that cluster I was enriched with tumors having papillary features and cluster III with tumors having squamous features, inspiring the labels "papillary" and "squamous," respectively [17]. As introduced above, a group based at the Broad Institute then repeated the exercise on an interim expanded cohort of 238 tumors and largely reproduced their original data [18]. They renamed the clusters "basal," "immune undifferentiated," "luminal immune," and "luminal" to reflect the dominant features they found in the gene expression signatures that defined each subtype [18].
Parallel efforts attempted to reconcile the molecular subtypes identified by the groups using shared whole transcriptome datasets [69-71]. The results of this work revealed a significant overlap among the subtype calls. The most upstream division occurred at the level of the basal versus luminal bifurcation proposed by the group at UNC [69-71]. The other subtypes appeared to mostly represent subdivisions of these subtypes. The MD Anderson p53-like subtype, which shared similarities with the Lund infiltrated subtype [65], TCGA's original cluster II [17], and the updated TCGA "immune undifferentiated" and "luminal immune" subtypes [18], consisted of a mixture of basal and luminal tumors that were heavily infiltrated with stromal cells, and the Lund uroA and GU tumors largely corresponded to UNC luminal tumors. Although the uroB tumors were assigned to the basal subtypes identified by other groups, the Lund group recognized that the uroB tumors contained FGFR3 pathway gene expression signatures and were enriched with activating FGFR3 mutations, and concluded that they probably corresponded to progressed uroA tumors [65].

Subtype membership had important implications for clinical outcomes. Similar to basal-like breast cancers [7], the Lund SCCL and uroB tumors and the squamous/basal tumors identified by the groups at UNC, MD Anderson Cancer Center, and TCGA were aggressive, and associated with advanced stage and metastatic disease at presentation, squamous histopathological features, and shorter survival in the absence of neoadjuvant cisplatin-based combination chemotherapy $[17,65,67,68,72]$. However, about half of basal tumors were downstaged by NAC [67], and early preliminary data suggested that the overall benefit provided by NAC might be greatest in patients whose tumors belonged to the basal molecular subtype [73]. If these preliminary data are confirmed in larger and more mature clinical datasets, the results would be reminiscent of past experience in breast cancer, where NAC has produced the greatest benefit in patients with highly proliferative basal-like (and HER2enriched) tumors $[8,12]$. Although the molecular mechanisms that underlie the benefit produced by chemotherapy in basal tumors are still under investigation, basal human bladder cancer cell lines are more sensitive to cisplatininduced apoptosis than are luminal cell lines (A. Ochoa, D.J. McConkey, unpublished observations). Conversely, NAC produced less clinical benefit in patients whose tumors belong to the infiltrated/p53-like subtype in the clinical trials that have been performed to date $[67,73]$.

The variable levels of immune cell infiltration observed in the bladder cancer subtypes [18] suggested that patients with these tumors might derive different amounts of benefit from immunotherapies [74]. Consistent with these predictions, early results suggest that TCGA subtype membership may be an independent predictor of benefit from therapy with the anti-PDL1 antibody atezolizumab [75]. In the phase II trial that led to Food and Drug Administration approval of the drug, patients whose tumors belonged to TCGA cluster II obtained somewhat more benefit than patients whose tumors belonged to the other subtypes, and patients with "papillary" (cluster I) tumors derived little benefit, if at all [75]. Importantly, immune 


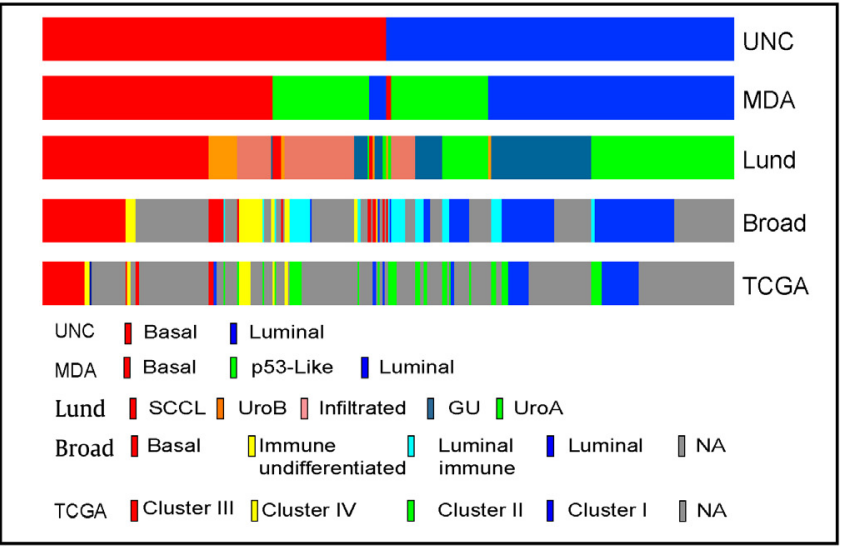

Lund
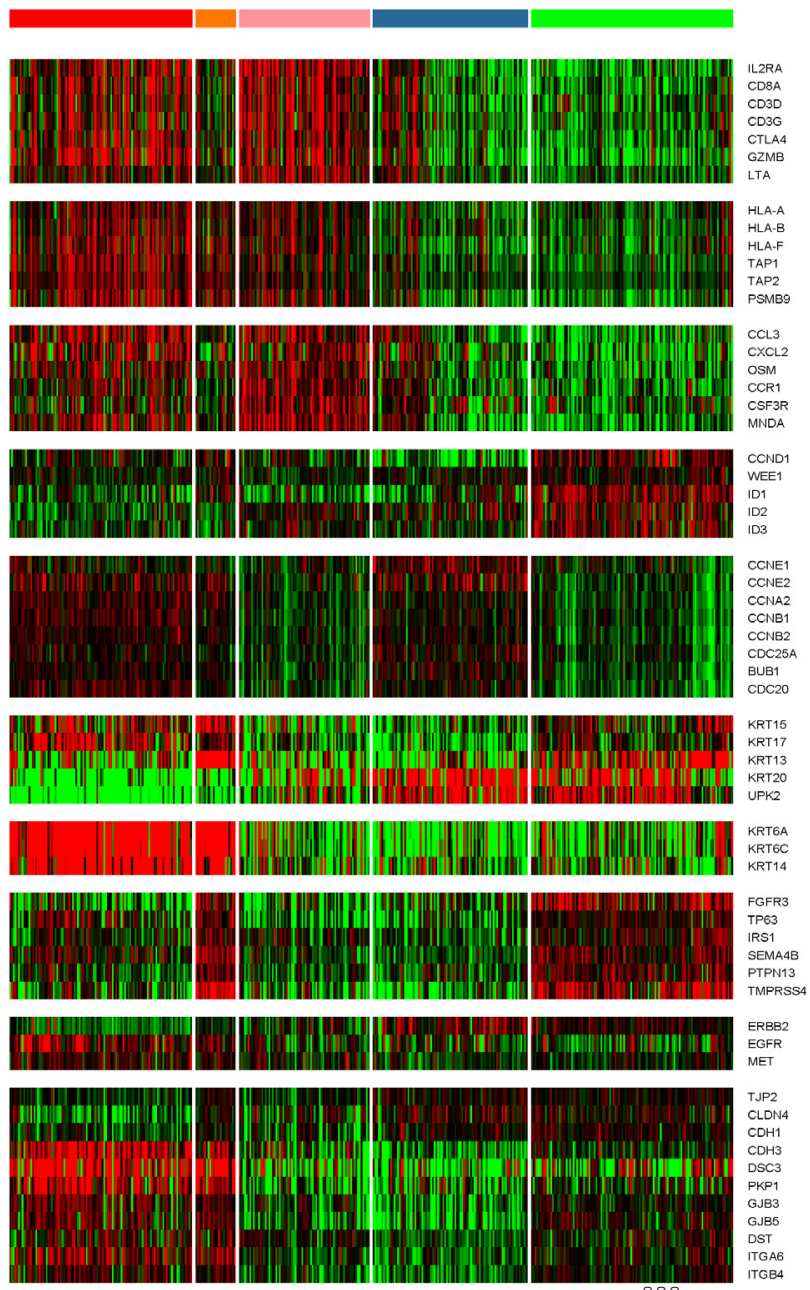

| SCCL \UroB \| Infiltrated | GU | UroA
UNC
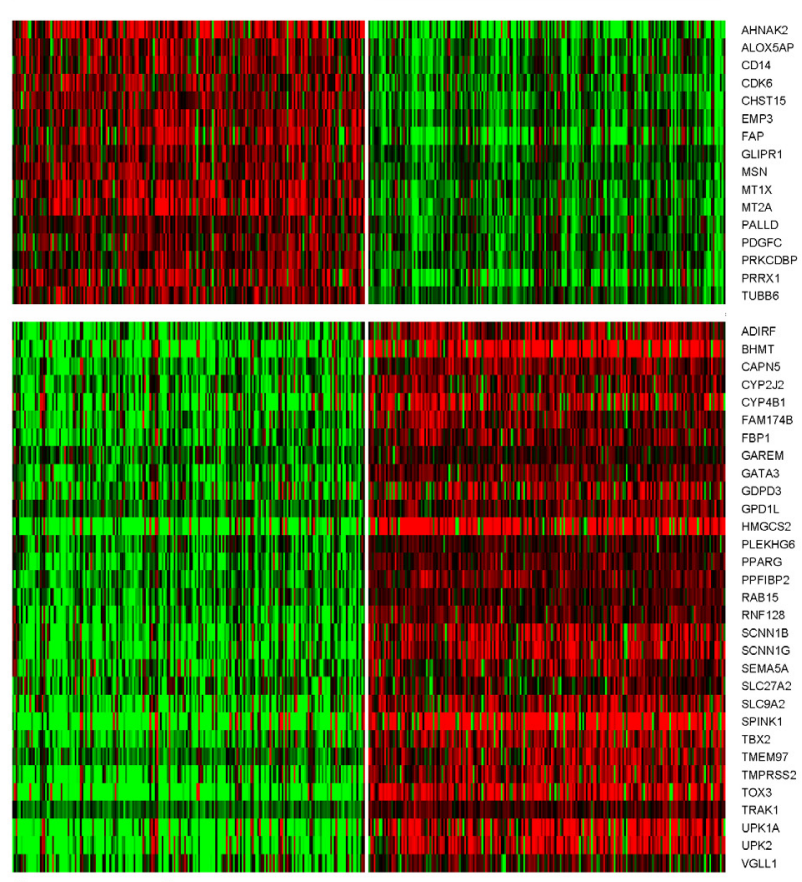

| Basal | Luminal

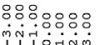

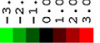

\section{MDA}
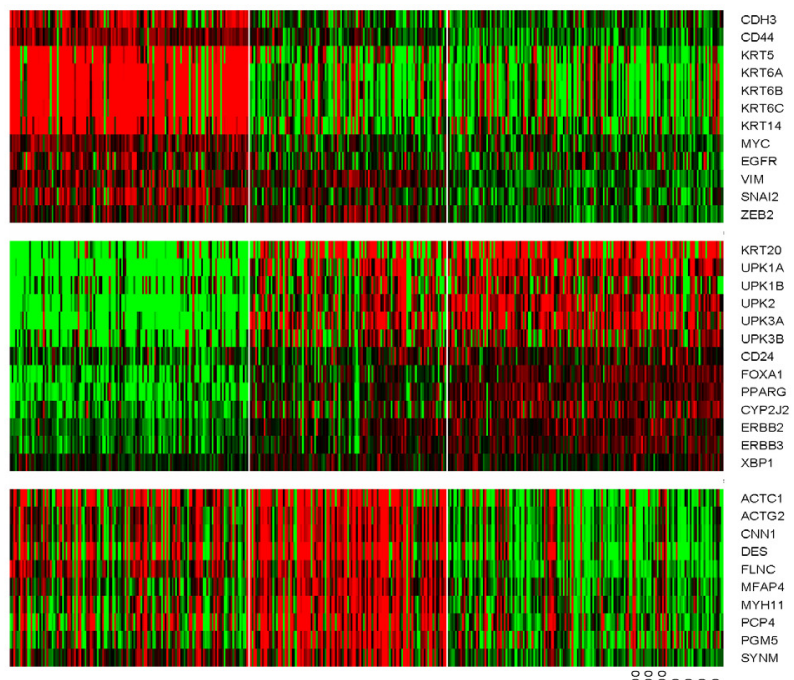

』Basal | p53-Like |Luminal

Fig. 1 - Comparison of subtype calls in TCGA's final dataset. Each group used TCGA's normalized RNA-seq data to assign TCGA's tumors to the UNC, MD Anderson, or Lund subtypes. Published calls made by a group at The Broad Institute [18] and TCGA were also included for comparison. The top left panel provides a schematic overview of the relationships among the calls made by the five groups. The heat maps display the relative expression of the gene sets that characterize each group's subtypes. The red and green colors correspond to high and low relative expression, respectively. GU = genomically unstable; MDA = MD Anderson; NA = not applicable; $\mathrm{SCCL}$ = squamous cell carcinoma like; TCGA = The Cancer Genome Atlas; UNC = University of North Carolina; uroA = urobasal A; uroB = urobasal B. 
infiltration and tumor PDL1 expression are actually highest in TCGA cluster IV, which corresponds to a subset of "mesenchymal" basal tumors [76], and this connection between EMT and immune infiltration and tumor PDL1 expression was observed across solid tumors in a recent pancancer analysis [77]. Therefore, even though TCGA cluster IV tumors are heavily infiltrated with lymphocytes, the T cells appear to be more actively suppressed than are the T cells in the tumors that belong to TCGA cluster II luminal subtype [76], which could explain why cluster IV tumors are somewhat less sensitive to immune checkpoint blockade. It could be noteworthy that atezolizumab provided maximal benefit in a portion of the tumors that belonged to the subtype that had been previously defined as being more resistant to conventional chemotherapy [67,73]. In other words, if the findings are validated, cisplatin-based chemotherapy and atezolizumab may produce clinical benefit in complementary populations of patients.

\subsection{Genomic alterations in molecular subtypes of MIBC}

Given past observations in the molecular subtypes in other cancers, it seemed likely that the molecular subtypes of bladder cancer would contain distinct mutations and CNAs. To test this hypothesis, we established a collaboration to assign the tumors from the complete TCGA RNA-seq dataset ( $n=408$ ) to subtypes using the classifiers developed at UNC, MD Anderson, and Lund University. We also obtained the subtype calls from the original TCGA marker paper [17] and the subsequent analyses performed by the group at the Broad Institute ( $n=238$ tumors) [18], in order to compare the calls with those made by our groups. We then examined each subtype for its content of specific DNA mutations ( $n=391$, available from Firehose [https://gdac. broadinstitute.org/]) and CNAs ( $n=404$, available from cBioportal [http://www.cbioportal.org/]). The results confirmed the patterns of subtype overlap noted in a recent study [69]. Specifically, the UNC basal-like subtype contained almost all the MD Anderson basal, Lund SCCL, and Broad basal tumors, and TCGA clusters III and IV (Fig. 1), strongly supporting the consensus view that the basal/SCClike subtype is consistently observed in muscle-invasive tumors [78]. The UNC basal-like subtype also contained half of the MD Anderson p53-like, most of the Lund uroB and infiltrated, and all the Broad immune undifferentiated tumors (Fig. 1). The UNC luminal subtype contained almost all the MD Anderson luminal tumors, half of the MD Anderson p53-like tumors, most of the Lund GU and uroA tumors, most of the Broad luminal immune and luminal tumors, and TCGA clusters I and II (Fig. 1).
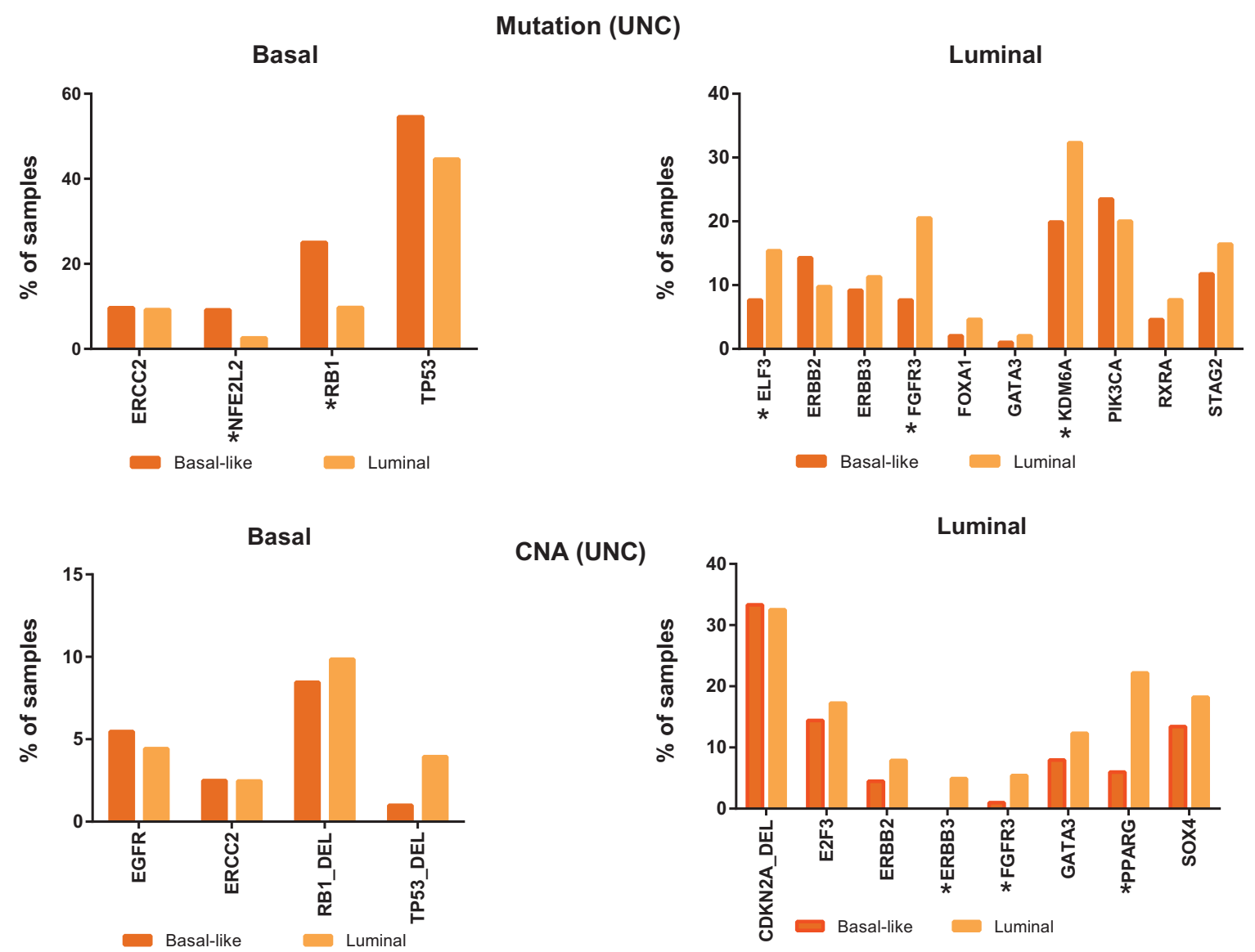

Fig. 2 - Enrichment of significantly mutated genes and CNAs in the UNC subtypes. Alterations are grouped according to predicted enrichment in basal versus luminal tumors, and the results are displayed as percentages of tumors in each subtype that contained the indicated alteration. The CNAs correspond to chromosomal amplification unless specifically identified as deletions ("del”). Fisher's exact test was used to determine differences between subtypes. CNA = copy number aberration; UNC = University of North Carolina. ${ }^{*} p<0.05$ was considered significant. 
We then used the top 30 most prevalent mutations and CNAs in TCGA's whole exome sequencing dataset in Firehose and cBioportal, respectively, and examined their prevalence in the UNC basal-like and luminal subtypes (Supplementary Fig. 1 and 2). Included among them were alterations that were enriched in the breast cancer intrinsic subtypes (TP53, RB1, ERBB2, and PIK3CA), genes that displayed different mutation frequencies in NMIBCs versus MIBCs (FGFR3, KDM6A, and STAG2), and genes that encode for mRNAs that were enriched in basal or luminal MIBCs (EGFR, PPARG, GATA3, ELF3, and ERBB3). Consistent with the overall hypothesis, several of the alterations were significantly enriched in either UNC basal-like or luminal cancers (Fig. 2).

We then investigated whether creating further subdivisions of the UNC molecular subtypes caused additional patterns of enrichment as had been documented previously [69]. Although the mutations and CNAs that were enriched in the UNC basal-like and luminal MIBCs were also enriched in the MD Anderson basal and luminal MIBCs, isolating the p53-like tumors did not further enhance enrichment (Fig. 3). Similarly, no mutations or CNAs were specifically enriched in the Lund infiltrated tumors as compared with the other Lund subtypes in this panel (Fig. 4). Therefore, it appears that the biology of these infiltrated tumors is dictated less by genetic influences than by other factors, such as the tumor microenvironment, explaining why their subtype membership was somewhat unstable [67,69]. On the other hand, subdividing the UNC basal-like and luminal tumors into the other Lund subtypes yielded additional and highly informative patterns of mutation and CNA enrichment. The uro $A$ and uroB tumors were both highly enriched with activating FGFR3 mutations [65,66,69], and the uroB tumors also contained a higher number of CDKN2A (p16) deletions (Fig. 4). The uroA and uroB tumors were also characterized by fewer RB1 mutations, and the uroB tumors could be distinguished from the uroA tumors by their content of PIK3CA, NFE2L2, ERBB2, and ERBB3 mutations (Fig. 4). Finally, the Lund subdivision of the UNC luminal MIBCs into the GU and uroA subtypes yielded additional informative patterns of mutation and CNA enrichment [69]. The GU tumors could be distinguished from the uroA tumors by the absence of activating FGFR3 mutations and by the presence of TP53 and ERCC2 mutations, RB1 deletions,

\section{Mutation (MDA)}
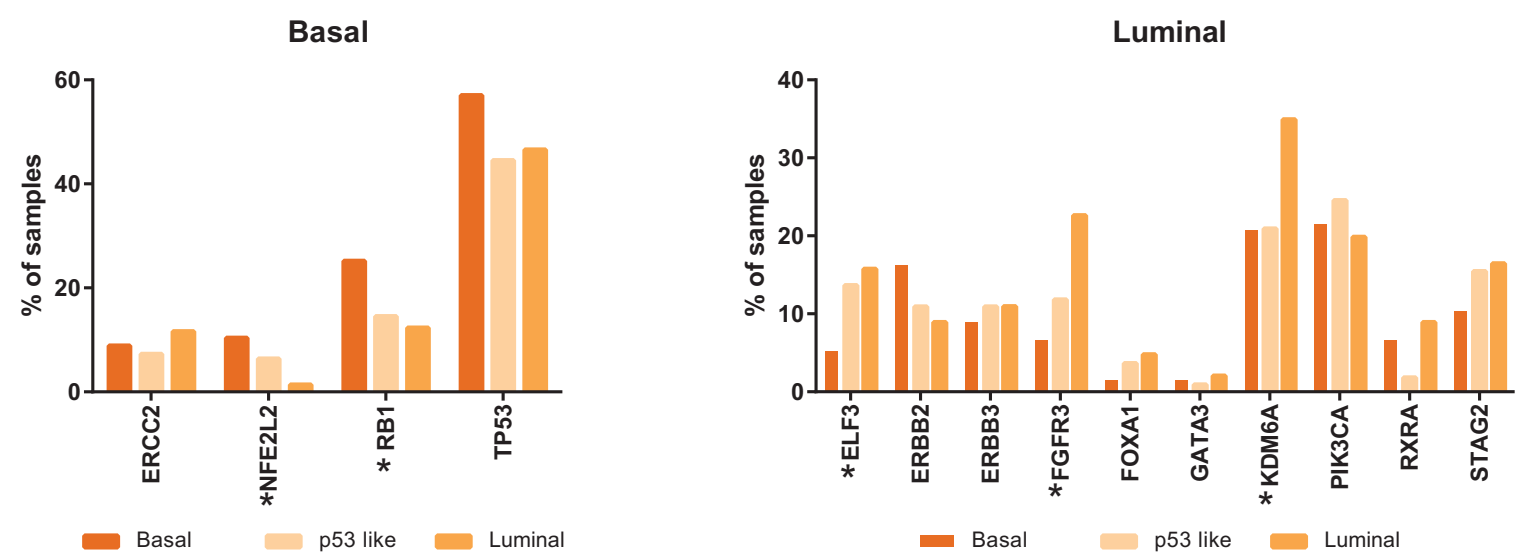

CNA (MDA)

Basal

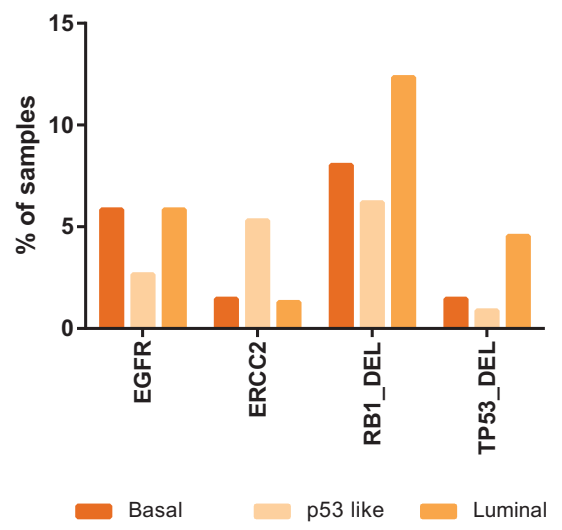

Luminal

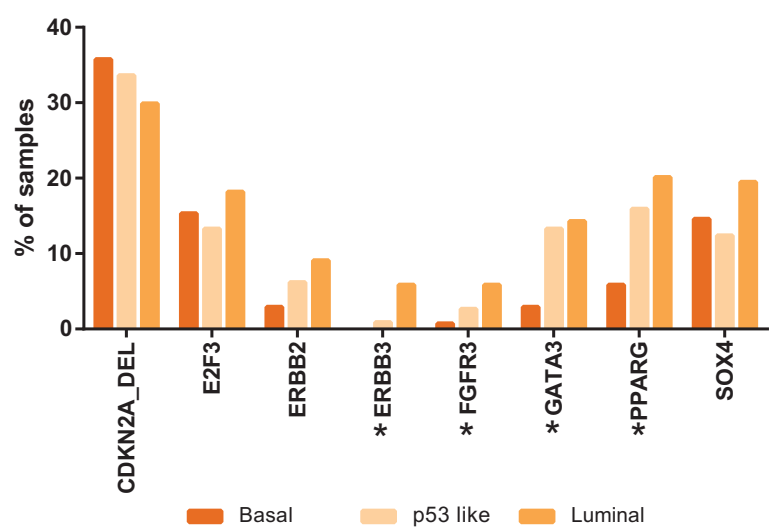

Fig. 3 - Enrichment of significantly mutated genes and CNAs in the MD Anderson subtypes. Alterations are grouped according to predicted enrichment in basal versus luminal tumors, and the results are displayed as percentages of tumors in each subtype that contained the indicated alteration. The CNAs correspond to chromosomal amplification unless specifically identified as deletions ("del"). Fisher's exact test was used to determine differences between subtypes. $\mathrm{CNA}=$ copy number aberration; $\mathrm{MDA}=\mathrm{MD}$ Anderson. ${ }^{*} p<0.05$ was considered significant. 


\section{Mutation (Lund)}
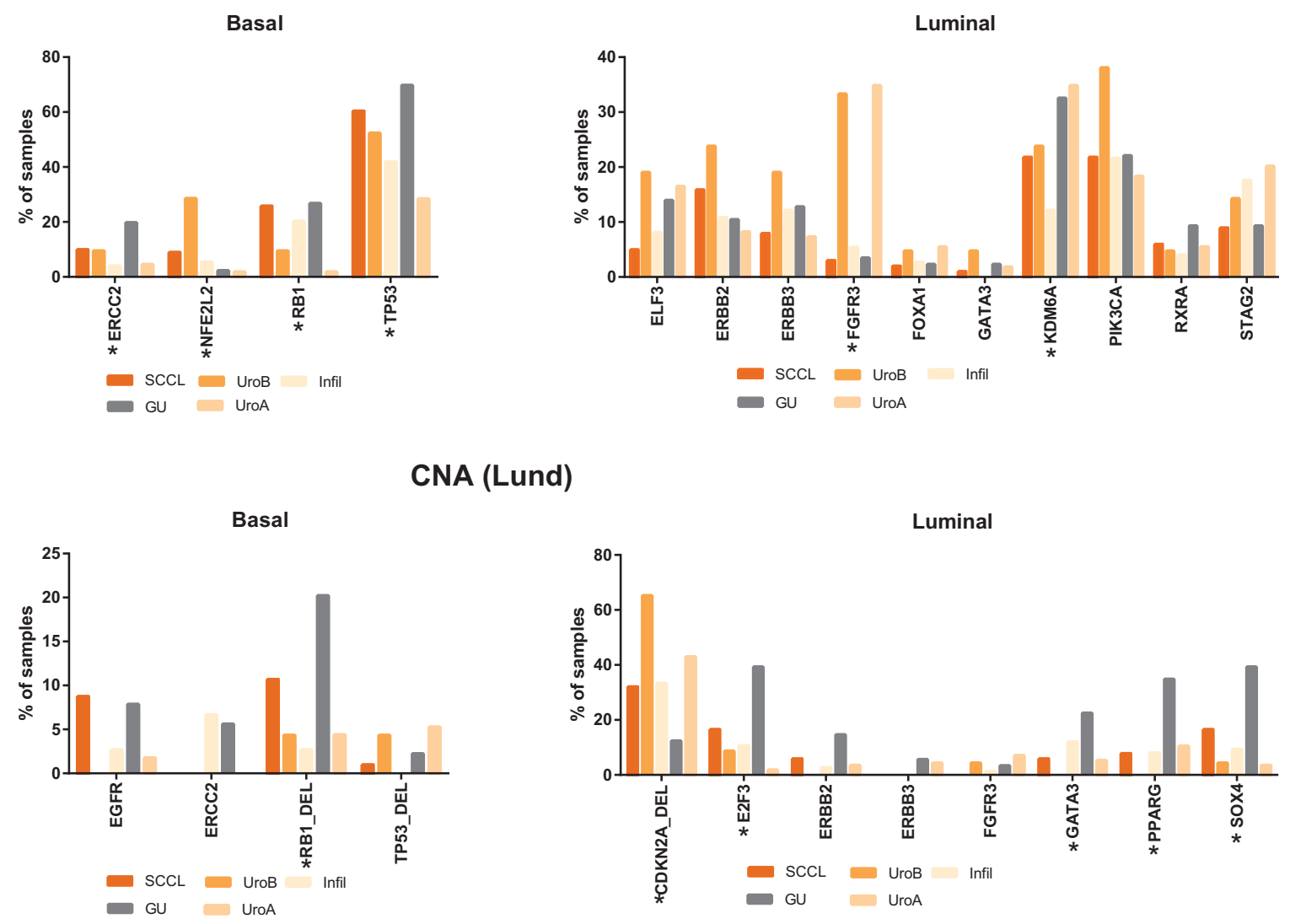

CNA (Lund)

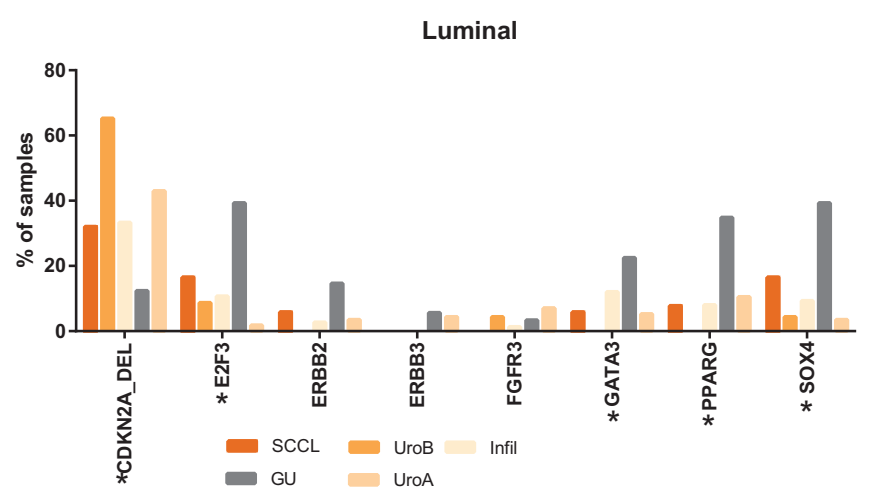

Fig. 4 - Enrichment of significantly mutated genes and CNAs in the Lund subtypes. Alterations are grouped according to predicted enrichment in basal versus luminal tumors, and the results are displayed as percentages of tumors in each subtype that contained the indicated alteration. The CNAs correspond to chromosomal amplification unless specifically identified as deletions (“del”). Fisher's exact test was used to determine differences between subtypes. $\mathrm{CNA}=$ copy number aberration; $\mathrm{GU}$ = genomically unstable; Infil = infiltrated; $S C C L=$ squamous cell carcinoma like; uroA = urobasal $A$; uroB $=$ urobasal $B .{ }^{*} p<0.05$ was considered significant.

and amplification of PPARG, GATA3, ERBB2, and E2F3/SOX4 (Fig. 4).

\section{Conclusions}

Molecular subtypes of bladder cancer are enriched with specific genetic alterations. As recognized previously [68], basal/SCC-like MIBCs frequently contain RB1 mutations, a property that they share with basal-like breast cancers [79,80]. Basal/SCC-like MIBCs are also enriched with NFE2L2 mutations, which have also been identified in lung and head and neck squamous cancers [21,22]. Luminal tumors contain more alterations in FGFR3 and KDM6A (also known as UTX) genes that are more commonly mutated in NMIBCs as compared with that in MIBCs [1]. These observations support the emerging conclusion that FGFR3 mutations mark the luminal MIBCs that correspond to the papillary NMIBCs that have progressed to become muscle invasive. Alterations affecting several transcription factors that appear to play important roles in urothelial terminal differentiation [81,82] (PPARG, GATA3, RXRA, and ELF3) were also enriched in luminal cancers. Biological effects of these alterations will need to be explored in future functional studies.

The Lund subclassifications divide the UNC/MD Anderson/TCGA basal/SCC-like and luminal subtypes in ways that have important biological and clinical implications. Although they cluster together with the squamous/basal tumors in the UNC, MD Anderson, and TCGA classifications, the genetic alterations in the uroB tumors more closely resemble those present in the luminal uroA subtype, supporting the conclusion that they represent progressed versions of the uroA cancers. The precise mechanisms that cause them to appear more "basal" (at the molecular level, and also in terms of their enrichment with squamous histological features and lethality) will be very interesting; their relatively high content of RB1 and NFE2L2 mutations suggests possible mechanisms. The existence of uroB tumors also suggests that basal versus luminal subtype class "switching" is possible. Clinically, it will be interesting to determine whether the uroA and uroB tumors are equally sensitive to FGFR inhibitors. 
The subdivision in the UNC/MD Anderson/TCGA luminal tumors that is created by the Lund classifier also appears to be extremely important. It is very interesting that the GU and uroA tumors are enriched with somewhat mutually exclusive patterns of mutations and CNAs involving key luminal genes (PPARG/GATA3 vs FGFR3). Overall, more of the top genes in the GU tumors were affected by CNAs than they were in the other molecular subtypes. The fact that GU tumors are enriched with ERCC2 mutations is also noteworthy. It will be interesting to determine their relationships to cigarette smoking [23] and relative sensitivities to NAC [35]. Given that ERCC2, RB1 [36], and ERBB2 [83] mutations and CNA levels in general [35] have been linked to chemosensitivity, it seems likely that patients with GU tumors will obtain greater direct clinical benefit from NAC than those who have uroA tumors.

It should be emphasized that our understanding of the biological and clinical properties of the molecular subtypes of bladder cancer is still fairly limited. Most of the available genomic and associated clinical data were obtained retrospectively, and the clinical follow-up is fairly short. Although the total number of profiled bladder cancers is increasing, it is relatively small, and challenges associated with merging the data that have been and continue to be generated on different genomic platforms make generating meta-datasets difficult. Preclinical studies implicating different cells of origin in the formation of papillary [84] and nonpapillary [56] cancers provide possible explanations for the origins of basal and luminal bladder cancers, but their relevance to human carcinogenesis remains unclear. The specific effects of most of the DNA alterations that have been identified in bladder cancers need to be explored much more deeply, presumably in preclinical models, to determine whether subtype context is important for their effects. The new information provided by TCGA and other groups will enable laboratory scientists to create models that more accurately capture important aspects of the genomic heterogeneity observed in patients.

We do not yet know whether molecular subtype membership is a stable, "intrinsic" feature of a given tumor. Bioinformatic analyses have already demonstrated that membership in the p53-like/infiltrated/TCGA cluster II subtype is relatively unstable, and we have demonstrated that luminal tumors often become p53-like after NAC [67]. These observations could explain why TCGA cluster II membership is not even more strongly associated with response to immune checkpoint blockade than has been observed in recently completed clinical trials [75]. In addition, as noted above, the uroB subtype may establish a precedent for luminal-to-basal subtype "switching" in bladder cancer. Muscle-invasive tumors can be multifocal, and our collaborators are currently performing wholeorgan mapping studies to determine whether all these multifocal tumors belong to the same subtype (B. Czerniak, personal communication). NMIBCs are prone to recurrence, and it will be important to perform longitudinal studies to determine how often subtype membership is maintained in these recurrences. Ongoing studies are performing deep genomic characterizations of metastases, and it will be interesting to see whether primary tumors and metastases always belong to the same subtype. Finally, additional comparisons of the DNA alterations in and subtype membership of tumors collected before and after neoadjuvant therapies, and where possible, systemic therapy for metastatic disease, must still be performed to determine whether subtype membership is stable. This information has important implications for prognostication and subtype-based therapy.

Author contributions: David J. McConkey had full access to all the data in the study and takes responsibility for the integrity of the data and the accuracy of the data analysis.

Study concept and design: Choi, Ochoa, McConkey. Acquisition of data: Choi, Aine, Höglund, Kim.

Analysis and interpretation of data: Choi, Ochoa, McConkey.

Drafting of the manuscript: Choi, Ochoa, McConkey.

Critical revision of the manuscript for important intellectual content: Aine, Höglund, Kim, Real, Kiltie, Lerner, Milsom, Dyrskjøt.

Statistical analysis: Choi, Ochoa.

Obtaining funding: McConkey.

Administrative, technical, or material support: None.

Supervision: McConkey.

Other: None.

Financial disclosures: David J. McConkey certifies that all conflicts of interest, including specific financial interests and relationships and affiliations relevant to the subject matter or materials discussed in the manuscript (eg, employment/affiliation, grants or funding, consultancies, honoraria, stock ownership or options, expert testimony, royalties, or patents filed, received, or pending), are the following: None.

Funding/Support and role of the sponsor: This work was supported by the MD Anderson Bladder SPORE (CA091846), the Cancer Prevention and Research Institute of Texas (CPRIT) (RP140542), and V foundation.

\section{Appendix A. Supplementary data}

Supplementary data associated with this article can be found, in the online version, at http://dx.doi.org/10.1016/j. eururo.2017.03.010.

\section{References}

[1] Knowles MA, Hurst CD. Molecular biology of bladder cancer: new insights into pathogenesis and clinical diversity. Nat Rev Cancer 2015;15:25-41.

[2] Dinney CP, McConkey DJ, Millikan RE, et al. Focus on bladder cancer. Cancer Cell 2004;6:111-6.

[3] Kamat AM, Hahn NM, Efstathiou JA, et al. Bladder cancer. Lancet 2016;388:2796-810.

[4] Golub TR, Slonim DK, Tamayo P, et al. Molecular classification of cancer: class discovery and class prediction by gene expression monitoring. Science 1999;286:531-7.

[5] Alizadeh AA, Eisen MB, Davis RE, et al. Distinct types of diffuse large B-cell lymphoma identified by gene expression profiling. Nature 2000;403:503-11.

[6] Perou CM, Sorlie T, Eisen MB, et al. Molecular portraits of human breast tumours. Nature 2000;406:747-52.

[7] Sorlie T, Perou CM, Tibshirani R, et al. Gene expression patterns of breast carcinomas distinguish tumor subclasses with clinical implications. Proc Natl Acad Sci U S A 2001;98:10869-74. 
[8] Prat A, Parker JS, Karginova O, et al. Phenotypic and molecular characterization of the Claudin-low intrinsic subtype of breast cancer. Breast Cancer Res 2010;12:R68.

[9] von Minckwitz G, Untch M, Blohmer JU, et al. Definition and impact of pathologic complete response on prognosis after neoadjuvant chemotherapy in various intrinsic breast cancer subtypes. J Clin Oncol 2012;30:1796-804.

[10] Slamon DJ, Leyland-Jones B, Shak S, et al. Use of chemotherapy plus a monoclonal antibody against HER2 for metastatic breast cancer that overexpresses HER2. N Engl J Med 2001;344:783-92.

[11] Prat A, Ellis MJ, Perou CM. Practical implications of gene-expression-based assays for breast oncologists. Nat Rev Clin Oncol 2012;9:48-57.

[12] von Minckwitz G, Eidtmann H, Rezai M, et al. Neoadjuvant chemotherapy and bevacizumab for HER2-negative breast cancer. N Engl J Med 2012;366:299-309.

[13] Chung CH, Parker JS, Karaca G, et al. Molecular classification of head and neck squamous cell carcinomas using patterns of gene expression. Cancer Cell 2004;5:489-500.

[14] Liang Y, Diehn M, Watson N, et al. Gene expression profiling reveals molecularly and clinically distinct subtypes of glioblastoma multiforme. Proc Natl Acad Sci U S A 2005;102:5814-9.

[15] Collisson EA, Sadanandam A, Olson P, et al. Subtypes of pancreatic ductal adenocarcinoma and their differing responses to therapy. Nat Med 2011;17:500-3.

[16] Hoadley KA, Yau C, Wolf DM, et al. Multiplatform analysis of 12 cancer types reveals molecular classification within and across tissues of origin. Cell 2014;158:929-44.

[17] The Cancer Genome Atlas Research Network. Comprehensive molecular characterization of urothelial bladder carcinoma. Nature 2014;507:315-22.

[18] Kim J, Akbani R, Creighton CJ, et al. Invasive bladder cancer: genomic insights and therapeutic promise. Clin Cancer Res 2015;21:4514-24.

[19] Hedegaard J, Lamy P, Nordentoft I, et al. Comprehensive transcriptional analysis of early-stage urothelial carcinoma. Cancer Cell 2016;30:27-42.

[20] Ito N, Hiasa Y, Tamai A, Okajima E, Kitamura H. Histogenesis of urinary bladder tumors induced by N-butyl-N-(4-hydroxybutyl)nitrosamine in rats. Gan 1969;60:401-10.

[21] The Cancer Genome Atlas Network. Comprehensive genomic characterization of head and neck squamous cell carcinomas. Nature 2015;517:576-82.

[22] The Cancer Genome Atlas Research Network. Comprehensive genomic characterization of squamous cell lung cancers. Nature 2012;489:519-25.

[23] Kim J, Mouw KW, Polak P, et al. Somatic ERCC2 mutations are associated with a distinct genomic signature in urothelial tumors. Nat Genet 2016;48:600-6.

[24] Swanton C, McGranahan N, Starrett GJ, Harris RS. APOBEC enzymes: mutagenic fuel for cancer evolution and heterogeneity. Cancer Discov 2015;5:704-12.

[25] Nordentoft I, Lamy P, Birkenkamp-Demtroder K, et al. Mutational context and diverse clonal development in early and late bladder cancer. Cell Rep 2014;7:1649-63.

[26] de Bruin EC, McGranahan N, Mitter R, et al. Spatial and temporal diversity in genomic instability processes defines lung cancer evolution. Science 2014;346:251-6.

[27] Gui Y, Guo G, Huang Y, et al. Frequent mutations of chromatin remodeling genes in transitional cell carcinoma of the bladder. Nat Genet 2011;43:875-8.

[28] Lindgren D, Frigyesi A, Gudjonsson S, et al. Combined gene expression and genomic profiling define two intrinsic molecular subtypes of urothelial carcinoma and gene signatures for molecular grading and outcome. Cancer Res 2010;70:3463-72.

[29] Sidransky D, Von Eschenbach A, Tsai YC, et al. Identification of p53 gene mutations in bladder cancers and urine samples. Science 1991;252:706-9.

[30] Spruck 3rd CH, Ohneseit PF, Gonzalez-Zulueta M, et al. Two molecular pathways to transitional cell carcinoma of the bladder. Cancer Res 1994;54:784-8.

[31] Lopez-Knowles E, Hernandez S, Kogevinas M, et al. The p53 pathway and outcome among patients with T1G3 bladder tumors. Clin Cancer Res 2006;12:6029-36.

[32] Eriksson P, Aine M, Sjodahl G, Staaf J, Lindgren D, Hoglund M. Detailed analysis of focal chromosome arm 1q and $6 \mathrm{p}$ amplifications in urothelial carcinoma reveals complex genomic events on $1 \mathrm{q}$, and SOX4 as a possible auxiliary target on 6p. PLoS One 2013;8:e67222.

[33] Sauter G, Carroll P, Moch H, et al. c-myc copy number gains in bladder cancer detected by fluorescence in situ hybridization. Am J Pathol 1995;146:1131-9.

[34] Balbas-Martinez C, Sagrera A, Carrillo-de-Santa-Pau E, et al. Recurrent inactivation of STAG2 in bladder cancer is not associated with aneuploidy. Nat Genet 2013;45:1464-9.

[35] Van Allen EM, Mouw KW, Kim P, et al. Somatic ERCC2 mutations correlate with cisplatin sensitivity in muscle-invasive urothelial carcinoma. Cancer Discov 2014;4:1140-53.

[36] Plimack ER, Dunbrack RL, Brennan TA, et al. Defects in DNA repair genes predict response to neoadjuvant cisplatin-based chemotherapy in muscle-invasive bladder cancer. Eur Urol 2015;68:959-67.

[37] Guo G, Sun X, Chen C, Wu S, Huang P, Li Z, et al. Whole-genome and whole-exome sequencing of bladder cancer identifies frequent alterations in genes involved in sister chromatid cohesion and segregation. Nat Genet 2013;45:1459-63.

[38] Razzak M. Bladder cancer: STAG2 in the spotlight-have genomic studies identified a plausible biomarker? Nat Rev Urol 2013;10: 675.

[39] Solomon DA, Kim JS, Bondaruk J, et al. Frequent truncating mutations of STAG2 in bladder cancer. Nat Genet 2013;45:1428-30.

[40] Taylor CF, Platt FM, Hurst CD, Thygesen HH, Knowles MA. Frequent inactivating mutations of STAG2 in bladder cancer are associated with low tumour grade and stage and inversely related to chromosomal copy number changes. Hum Mol Genet 2014;23: 1964-74.

[41] Allory Y, Beukers W, Sagrera A, et al. Telomerase reverse transcriptase promoter mutations in bladder cancer: high frequency across stages, detection in urine, and lack of association with outcome. Eur Urol 2014;65:360-6.

[42] Borah S, Xi L, Zaug AJ, et al. Cancer. TERT promoter mutations and telomerase reactivation in urothelial cancer. Science 2015;347: 1006-10.

[43] Huang FW, Hodis E, Xu MJ, Kryukov GV, Chin L, Garraway LA. Highly recurrent TERT promoter mutations in human melanoma. Science 2013;339:957-9.

[44] Hurst CD, Platt FM, Knowles MA. Comprehensive mutation analysis of the TERT promoter in bladder cancer and detection of mutations in voided urine. Eur Urol 2014;65:367-9.

[45] Kinde I, Munari E, Faraj SF, et al. TERT promoter mutations occur early in urothelial neoplasia and are biomarkers of early disease and disease recurrence in urine. Cancer Res 2013;73:7162-7.

[46] Nickerson ML, Dancik GM, Im KM, et al. Concurrent alterations in TERT, KDM6A, and the BRCA pathway in bladder cancer. Clin Cancer Res 2014;20:4935-48.

[47] Rachakonda PS, Hosen I, de Verdier PJ, et al. TERT promoter mutations in bladder cancer affect patient survival and disease 
recurrence through modification by a common polymorphism. Proc Natl Acad Sci U S A 2013;110:17426-31.

[48] Vinagre J, Almeida A, Populo H, et al. Frequency of TERT promoter mutations in human cancers. Nat Commun 2013;4:2185.

[49] Cappellen D, De Oliveira C, Ricol D, et al. Frequent activating mutations of FGFR3 in human bladder and cervix carcinomas. Nat Genet 1999;23:18-20.

[50] van Rhijn BW, Lurkin I, Radvanyi F, Kirkels WJ, van der Kwast TH, Zwarthoff EC. The fibroblast growth factor receptor 3 (FGFR3) mutation is a strong indicator of superficial bladder cancer with low recurrence rate. Cancer Res 2001;61:1265-8.

[51] Lamont FR, Tomlinson DC, Cooper PA, Shnyder SD, Chester JD, Knowles MA. Small molecule FGF receptor inhibitors block FGFRdependent urothelial carcinoma growth in vitro and in vivo. $\mathrm{Br} \mathrm{J}$ Cancer 2011;104:75-82.

[52] di Martino E, L'Hote CG, Kennedy W, Tomlinson DC, Knowles MA. Mutant fibroblast growth factor receptor 3 induces intracellular signaling and cellular transformation in a cell type- and mutationspecific manner. Oncogene 2009;28:4306-16.

[53] Williams SV, Hurst CD, Knowles MA. Oncogenic FGFR3 gene fusions in bladder cancer. Hum Mol Gen 2013;22:795-803.

[54] Rampias T, Vgenopoulou P, Avgeris M, et al. A new tumor suppressor role for the Notch pathway in bladder cancer. Nat Med 2014;20:1199-205.

[55] Maraver A, Fernandez-Marcos PJ, Cash TP, et al. NOTCH pathway inactivation promotes bladder cancer progression. J Clin Invest 2015;125:824-30.

[56] Shin K, Lim A, Odegaard JI, et al. Cellular origin of bladder neoplasia and tissue dynamics of its progression to invasive carcinoma. Nat Cell Biol 2014;16:469-78.

[57] Shin K, Lim A, Zhao C, et al. Hedgehog signaling restrains bladder cancer progression by eliciting stromal production of urothelial differentiation factors. Cancer Cell 2014;26:521-33.

[58] Dyrskjot L, Thykjaer T, Kruhoffer M, et al. Identifying distinct classes of bladder carcinoma using microarrays. Nat Genet 2003;33:90-6.

[59] Blaveri E, Simko JP, Korkola JE, et al. Bladder cancer outcome and subtype classification by gene expression. Clin Cancer Res 2005;11:4044-55.

[60] Sanchez-Carbayo M, Socci ND, Lozano J, Saint F, Cordon-Cardo C. Defining molecular profiles of poor outcome in patients with invasive bladder cancer using oligonucleotide microarrays. J Clin Oncol 2006;24:778-89.

[61] Dyrskjot L, Kruhoffer M, Thykjaer T, et al. Gene expression in the urinary bladder: a common carcinoma in situ gene expression signature exists disregarding histopathological classification. Cancer Res 2004;64:4040-8.

[62] Dyrskjot L, Zieger K, Kruhoffer M, et al. A molecular signature in superficial bladder carcinoma predicts clinical outcome. Clin Cancer Res 2005;11:4029-36.

[63] Dyrskjot L, Zieger K, Real FX, et al. Gene expression signatures predict outcome in non-muscle-invasive bladder carcinoma: a multicenter validation study. Clin Cancer Res 2007;13:3545-51.

[64] Patschan O, Sjodahl G, Chebil G, et al. A molecular pathologic framework for risk stratification of stage $\mathrm{T} 1$ urothelial carcinoma. Eur Urol 2015;68:824-32, discussion 35-6.

[65] Sjodahl G, Lauss M, Lovgren K, et al. A molecular taxonomy for urothelial carcinoma. Clin Cancer Res 2012;18:3377-86.

[66] Sjodahl G, Lovgren K, Lauss M, et al. Toward a molecular pathologic classification of urothelial carcinoma. Am J Pathol 2013;183:681-91.

[67] Choi W, Porten S, Kim S, et al. Identification of distinct basal and luminal subtypes of muscle-invasive bladder cancer with different sensitivities to frontline chemotherapy. Cancer Cell 2014;25: 152-65.

[68] Damrauer JS, Hoadley KA, Chism DD, et al. Intrinsic subtypes of high-grade bladder cancer reflect the hallmarks of breast cancer biology. Proc Natl Acad Sci U S A 2014;111:3110-5.

[69] Aine M, Eriksson P, Liedberg F, Sjodahl G, Hoglund M. Biological determinants of bladder cancer gene expression subtypes. Sci Rep 2015;5:10957.

[70] Choi W, Czerniak B, Ochoa A, et al. Intrinsic basal and luminal subtypes of muscle-invasive bladder cancer. Nat Rev Urol 2014;11:400-10.

[71] McConkey DJ, Choi W, Dinney CP. New insights into subtypes of invasive bladder cancer: considerations of the clinician. Eur Urol 2014;66:609-10.

[72] Rebouissou S, Bernard-Pierrot I, de Reynies A, et al. EGFR as a potential therapeutic target for a subset of muscle-invasive bladder cancers presenting a basal-like phenotype. Sci Transl Med 2014;6:244ra91.

[73] McConkey DJ, Choi W, Shen Y, et al. A prognostic gene expression signature in the molecular classification of chemotherapynaive urothelial cancer is predictive of clinical outcomes from neoadjuvant chemotherapy: a phase 2 trial of dose-dense methotrexate, vinblastine, doxorubicin, and cisplatin with bevacizumab in urothelial cancer. Eur Urol 2016;69:855-62.

[74] McConkey DJ, Choi W, Ochoa A, Siefker-Radtke A, Czerniak B, Dinney CP. Therapeutic opportunities in the intrinsic subtypes of muscle-invasive bladder cancer. Hematol Oncol Clin North Am 2015;29:377-94, x-xi.

[75] Rosenberg JE, Hoffman-Censits J, Powles T, et al. Atezolizumab in patients with locally advanced and metastatic urothelial carcinoma who have progressed following treatment with platinum-based chemotherapy: a single-arm, multicentre, phase 2 trial. Lancet 2016;387:1909-20.

[76] Kardos J, Chai S, Mose LE, et al. Claudin-low bladder tumors are immune infiltrated and actively immune suppressed. JCI Insight 2016;1:e85902.

[77] Mak MP, Tong P, Diao L, et al. A patient-derived, pan-cancer EMT signature identifies global molecular alterations and immune target enrichment following epithelial-to-mesenchymal transition. Clin Cancer Res 2016;22:609-20.

[78] Lerner SP, McConkey DJ, Hoadley KA, et al. Bladder cancer molecular taxonomy: summary from a consensus meeting. Bladder Cancer 2016;2:37-47.

[79] Ellis MJ, Perou CM. The genomic landscape of breast cancer as a therapeutic roadmap. Cancer Discov 2013;3:27-34.

[80] The Cancer Genome Atlas Network. Comprehensive molecular portraits of human breast tumours. Nature 2012;490:61-70.

[81] Bock M, Hinley J, Schmitt C, Wahlicht T, Kramer S, Southgate J. Identification of ELF3 as an early transcriptional regulator of human urothelium. Dev Biol 2014;386:321-30.

[82] Varley CL, Stahlschmidt J, Lee WC, et al. Role of PPARgamma and EGFR signalling in the urothelial terminal differentiation programme. J Cell Sci 2004;117:2029-36.

[83] Groenendijk FH, de Jong J, Fransen van de Putte EE, et al. ERBB2 mutations characterize a subgroup of muscle-invasive bladder cancers with excellent response to neoadjuvant chemotherapy. Eur Urol 2016;69:384-8.

[84] Van Batavia J, Yamany T, Molotkov A, et al. Bladder cancers arise from distinct urothelial sub-populations. Nat Cell Biol 2014;16:982-91, 1-5. 